\title{
Neural breathing patterns in preterm newborns supported with non-invasive neurally adjusted ventilatory assist
}

\author{
Fermín García-Muñoz Rodrigo ${ }^{1}$ - Lourdes Urquía Martí ${ }^{1}$ - Gloria Galán Henríquez ${ }^{1}$ Sonia Rivero Rodríguez ${ }^{1}$. \\ Alberto Hernández Gómez ${ }^{2}$
}

Received: 27 January 2018 / Revised: 22 April 2018 / Accepted: 17 May 2018 / Published online: 18 June 2018

(c) Nature America, Inc., part of Springer Nature 2018

\begin{abstract}
Objective To characterize the neural breathing pattern in preterm infants supported with non-invasive neurally adjusted ventilatory assist (NIV-NAVA).

Study Design Single-center prospective observational study. The electrical activity of the diaphragm (EAdi) was periodically recorded in 30-second series with the Edi catheter and the Servo-n software (Maquet, Solna, Sweden) in preterm infants

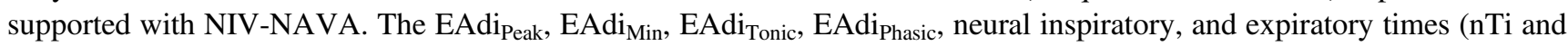
$\mathrm{nTe}$ ) and the neural respiratory rate (nRR) were calculated. EAdi curves were generated by Excel for visual examination and classified according to the predominant pattern.

Results 291 observations were analyzed in 19 patients with a mean GA of 27.3 weeks (range 24-36 weeks), birth weight

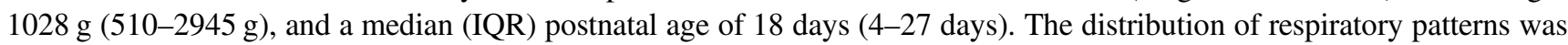
phasic without tonic activity $61.9 \%$, phasic with basal tonic activity 18.6 , tonic burst $3.8 \%$, central apnea $7.9 \%$, and mixed pattern $7.9 \%$. In addition, $12 \%$ of the records showed apneas of $>10$ seconds, and $50.2 \%$ one or more "sighs", defined as breaths with an EAdi $\mathrm{i}_{\text {Peak }}$ and/or nTi greater than twice the average EAdi Peak $_{\text {end/or }} \mathrm{nTi}$ of the recording. Neural times were measurable in 252 observations. The nTi was, median (IQR): $279 \mathrm{~ms}$ (253-285 ms), the nTe $764 \mathrm{~ms}$ (642-925 ms), and the nRR 63 bpm (51-70), with a great intra and inter-subjects variability.

Conclusions The neural breathing patterns in preterm infants supported with NIV-NAVA are quite variable and are characterized by the presence of significant tonic activity. Central apneas and sighs are common in this group of patients. The nTi seems to be shorter than the mechanical Ti commonly used in assisted ventilation.
\end{abstract}

\section{Introduction}

Several neural respiratory patterns have been described in spontaneously breathing preterm infants, from a regular phasic pattern to irregular rhythms with increased basal tone, tonic bursts, sighs, periodic breathing, and apneas of variable duration [1]. A specially designed catheter, the Edi catheter, which has 10 electrodes embedded in its wall that record the electrical activity of the diaphragm (EAdi), is used to feed the

Fermín García-Muñoz Rodrigo

fgarciamu@gmail.com

1 Neonatal Unit, Complejo Hospitalario Universitario Insular Materno-Infantil de Las Palmas de Gran Canaria, Avenida Marítima del Sur S/N, 35016 Las Palmas de Gran Canaria, Spain

2 Informática de sistemas (Computer Systems), Madrid, Spain patient and, in the neurally adjusted modalities, neurally adjusted ventilatory assist (NAVA) and non-invasive neurally adjusted ventilatory assist (NIV-NAVA), to synchronize respiratory support with patient efforts. This catheter also allows continuous monitoring of the EAdi in a minimally invasive way, which is useful for performing physiological measurements [2]. The maximum electrical activity (EAdiPeak) after neuromuscular coupling correlates with the intensity of the diaphragmatic contraction, which, in turn, correlates with the inspired tidal volume $\left(\mathrm{V}_{\mathrm{T}}\right)$. The EAdi is independent of air leaks during non-invasive ventilation (NIV), potentially offering an advantage for valid physiological measurements. The tonic EAdi ( EAdi $_{\text {Tonic }}$ ) involves the maintenance of diaphragmatic contraction during expiration, which is thought to contribute to the maintenance of the lung's functional residual capacity (FRC) [3-5].

To our knowledge, the neural respiratory patterns in preterm newborns assisted with NIV have not been 
Fig. 1 Electrical activity of diaphragm (EAdi) curve and parameters studied

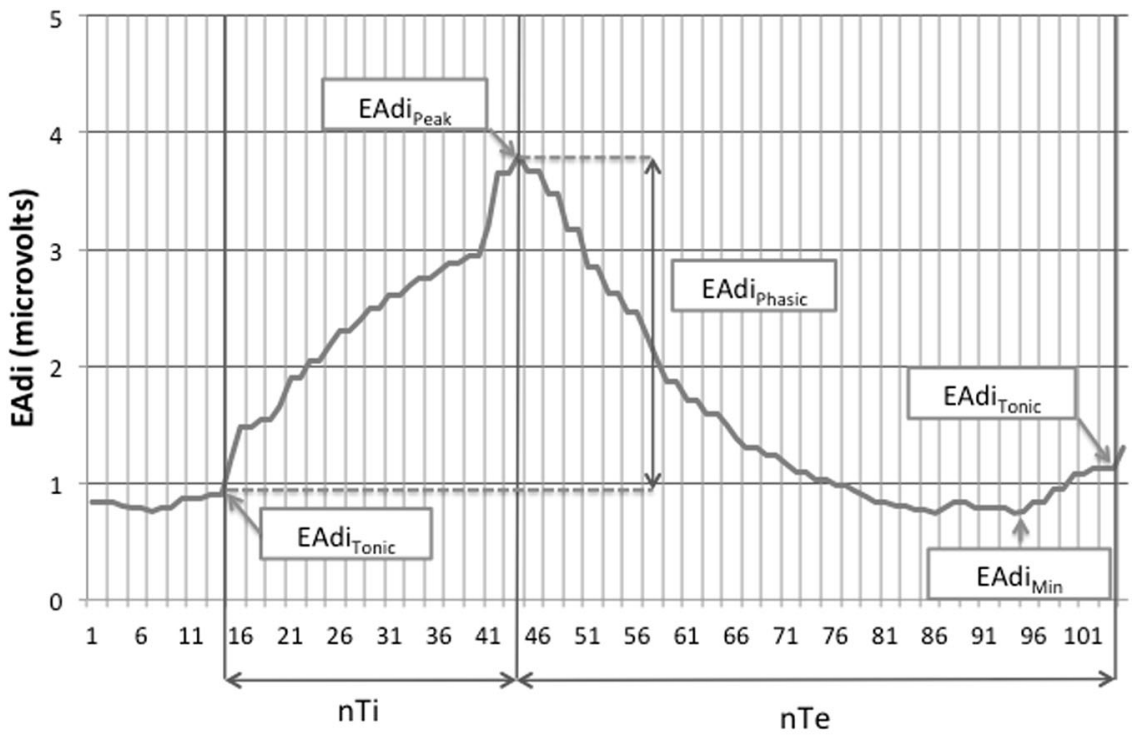

Time (hundredths of a second) systematically studied. The aim of our study was to characterize the neural breathing patterns in preterm neonates with mild to moderate respiratory disease supported with NIV-NAVA.

\section{Methods}

A prospective, observational, single-center study was conducted in a tertiary hospital with a third-level NICU in which NIV-NAVA is used as the standard non-invasive ventilatory support method at the discretion of the attending physician in charge of the patient. During the 10-month study period (April 2016 to January 2017), 19 consecutive preterm infants who received NIV-NAVA during hospitalization were included. NIV-NAVA was applied by means of nasal prongs or mask (Miniflow, Medin, Germany). The respiratory support was established and modified according to the patient's clinical needs. The NAVA level was set and periodically adjusted to maintain an EAdi $_{\text {Peak }}$ between 8 and 15 microvolts $(\mu \mathrm{V})$. The PEEP was set between 4 and 8 $\mathrm{cmH}_{2} \mathrm{O}$, with the aim of achieving adequate lung recruitment and an $\mathrm{EAdi}_{\mathrm{Min}}<5 \mu \mathrm{V}$. The upper limit of pressure was set according to the patient's GA between 30 and 40 $\mathrm{cmH}_{2} \mathrm{O}$, to allow proportional support during sighs, and the apnea time was set between 2 and 5 seconds.

The EAdi was periodically recorded using an orogastric tube, the Edi catheter, and the NAVA software that is incorporated into the SERVO-n equipment (Maquet, Solna, Sweden), which allows the recording of several parameters (EAdi, flow, pressure and volume) in 30-second series. At the time of the study, none of the patients received sedation, pharmacological analgesia, or muscle relaxants. Caffeine was administered to all patients less than 34 weeks gestational age (GA) who needed ventilatory support or were at risk for apnea according to our protocol. They had not been manipulated or fed in the previous 30 minutes and were in the supine position, either asleep or quietly alert. Before proceeding with recording the EAdi signal, the proper positioning of the catheter and its electrodes at the level of the esophagogastric junction (crural diaphragm) [6] was verified using the equipment software according to the manufacturer's recommendations. The measurements were carried out every 6 to $12 \mathrm{~h}$ in each patient, over several consecutive days.

The recordings were exported in ".txt" files for later analysis with a customized program. For the purpose of this investigation, a neurobreath was defined as a continuous increase of $>0.3 \mu \mathrm{V}$ for more than 80 milliseconds (ms). In each 30-second record, the average of the following parameters were calculated (Fig. 1): EAdi ${ }_{\text {Peak }}$ (maximum electrical activity of the diaphragm in each respiratory cycle); EAdi $_{\text {Min }}$ (minimum electrical activity during expiration); EAdi $_{\text {Tonic }}$ (baseline diaphragmatic electrical activity at end expiration, immediately before the beginning of the next neural inspiration); EAdi $_{\text {Phasic }}\left(\right.$ EAdi $\left._{\text {Peak }}-\mathrm{EAdi}_{\text {Tonic }}\right)$; neural inspiratory time (nTi, the time elapsed between the EAdi-

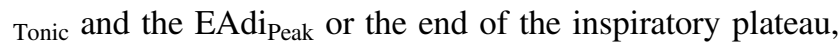
when present, of each breathing cycle); neural expiratory time (nTe, the time elapsed between the EAdi Peak $_{\text {or the end }}$ of the inspiratory plateau and the beginning of the next inspiratory cycle); neural total breathing time (nTbt, nTi + $\mathrm{nTe}$ ); and neural respiratory rate (nRR, calculated as 60/ $\mathrm{nTbt}$ ). EAdi was expressed in $\mu \mathrm{V}$ and time in ms. Breath-tobreath variability was studied by the coefficient of variation 


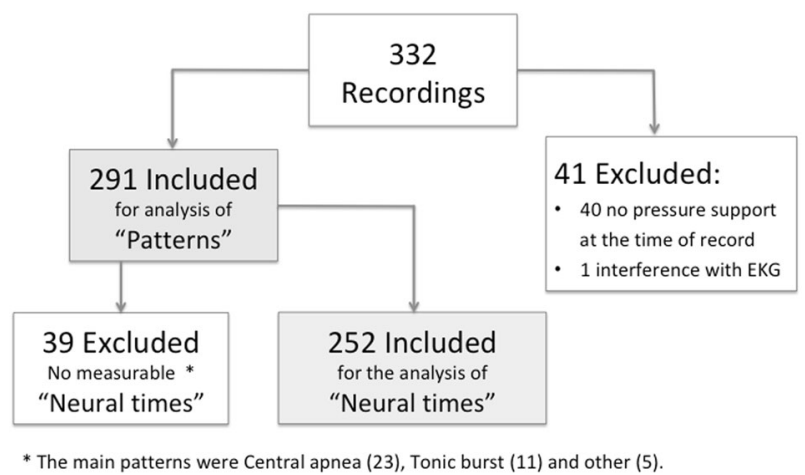

Fig. 2 Flow diagram of included and excluded recordings for analysis of breathing patterns and neural respiratory times

$((\mathrm{CV}=\mathrm{SD} / \mathrm{Mean}) \times 100)$ for each parameter, within subject and between subjects.

EAdi curves were generated by Excel for visual examination and each recording was classified according to the predominant pattern (i.e., the pattern present for more than $50 \%$ of the recording). A phasic breathing pattern was defined as occurring when a regular increase and decrease of the EAdi was clearly visualized on the graph and the nTi was shorter than $1 \mathrm{~s}$. Tonic activity was considered to be present when the EAdi waveform did not return to baseline and the value of the EAdi ${ }_{\text {Min }}$ was $>4 \mu \mathrm{V}$. Tonic bursts were defined as elevated tonic activity without clear, or with very irregular, phasic activity. Sighs were defined as any single breath with an EAdi ${ }_{\text {Peak }}$ and/or an nTi greater than twice the

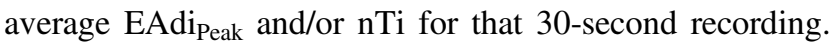
Finally, central apneas were defined as the presence of a continuous EAdi $<2 \mu \mathrm{V}$ for more than $5 \mathrm{~s}$, without phasic or tonic activity, and were classified by duration into apneas of $5-10$ seconds or $>10 \mathrm{~s}$. If more than one pattern was observed without a clear predominance of one over the others, the record was classified as a mixed pattern. Two investigators (FG-MR and LUM) independently assessed and classified the recordings. In the case of discrepancy, the definitive pattern was assigned by consensus after reevaluation by the two researchers.

Statistical analyses were performed with the SPSS software, version 20 (IBM Corp., Armonk, NY, USA). Continuous variables are expressed in the form of mean (standard deviation (SD)) or median (interquartile range (IQR)), and qualitative variables are expressed as proportions (\%). The study was approved by the Teaching, Research and Continued Training Board of the center, and verbal consent was obtained from the patients' parents before anonymous data collection.

\section{Results}

Figure 2 shows the study flow diagram with the total number of recordings obtained and the number of them suitable for the "Pattern" and "Neural times" analysis. A total of 332 recordings were obtained from 19 patients, with a median of $10(6-15)$ observations per patient. The principal characteristics of the patients are shown in Table 1. The mean GA was 27.3 weeks (range 24-36 weeks), and the mean birth weight was $1028 \mathrm{~g}$ (range 510-2945 g). The median (IQR) age at the time of the study was 18 (4-27) days, and the postmenstrual age (PMA) was 29 (28-31) weeks. Of the 332 available observations, 40 were recorded while the patient was breathing spontaneously without pressure support (during withdrawal attempts after weaning) and another one exhibited interferences with the cardiac rhythm, so they were excluded. In total, 291 recordings were included in the analysis of breathing patterns. The median ventilatory support was NAVA level $1(0.8-1) \mathrm{cm}$ $\mathrm{H}_{2} \mathrm{O} / \mu \mathrm{V}$, PEEP $6 \quad(5-6.5) \mathrm{cm} \quad \mathrm{H}_{2} \mathrm{O}$ and $\mathrm{FiO}_{2} 0.24$ (0.21-0.27). Figure 3 shows the observed breathing patterns. The most frequent respiratory pattern was phasic without tonic activity $(61.9 \%)$, followed by phasic with baseline tonic activity (18.6\%), central apnea (7.9\%), and tonic burst $(3.8 \%)$. Mixed patterns were present in $7.9 \%$ of the recordings. In addition, $13.4 \%$ and $12 \%$ of the recordings exhibited apneas of $5-10$ s or $>10$ s duration, respectively. Finally, $50.2 \%$ of the recordings showed one or more sighs. We did not find significant differences in the distribution of respiratory patterns in relation to the GA, birth weight, or postnatal age in this group of patients.

EAdi and Neural times were measurable in 252 recordings in which the breathing activity was predominantly phasic or mixed. The mean, SD, and CV of each parameter was calculated individually in each patient. Subsequently, the medians (IQR) of these values were computed for the whole group of patients (Table 2). The nTi and nTe were $279 \mathrm{~ms}(253-285 \mathrm{~ms})$ and $764 \mathrm{~ms}$ (642-925 ms), respectively, and the nRR was $63 \mathrm{bpm}(51-70 \mathrm{bpm})$. We did not find any correlation between the nTi and the GA, the chronological age or the PMA.

\section{Discussion}

Our study shows that neural respiratory patterns in preterm infants supported with NIV-NAVA are quite variable and are characterized by the presence of increased tonic activity during a significant proportion of the respiratory cycles. Furthermore, central apneas and sighs are common in this group of patients. Similar findings have been shown by Beck et al. [1] in spontaneously breathing preterm infants, although our patients were more immature, with an 


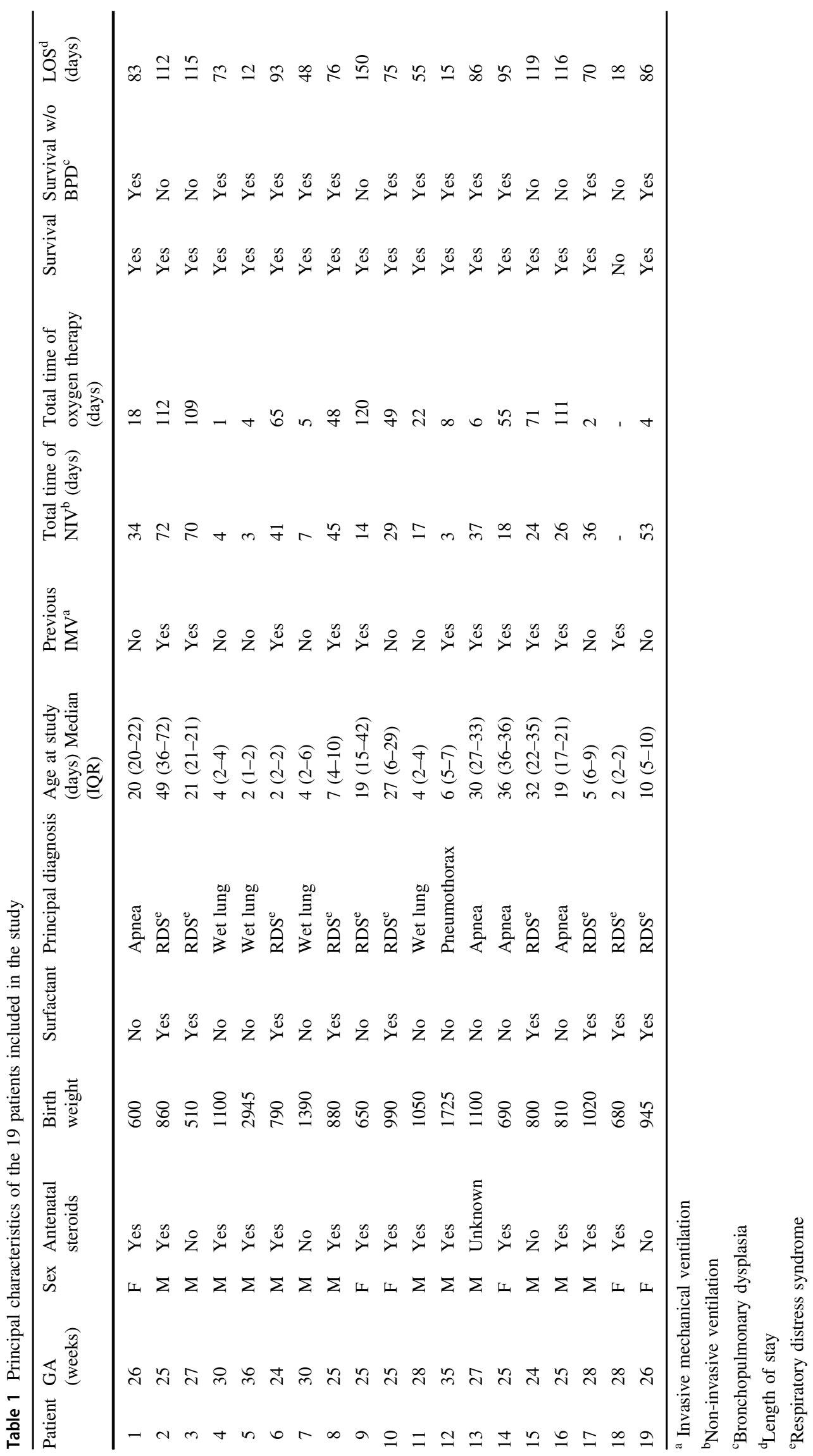




\section{Phasic without tonic activity.}

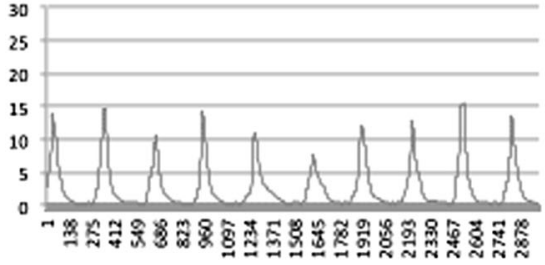

3. Tonic burst

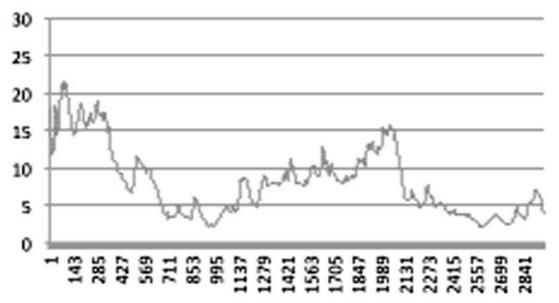

2. Phasic with tonic activity.

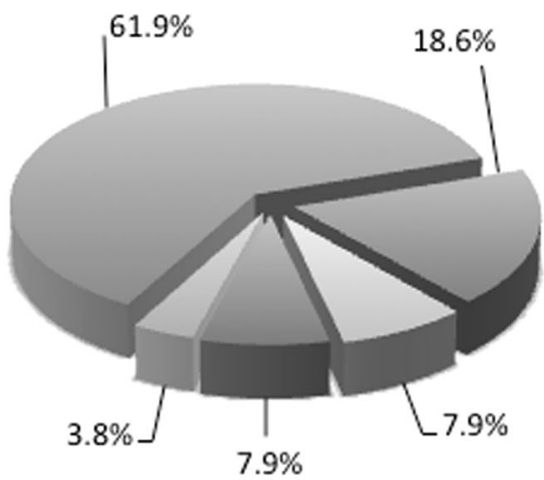

5. Mixed pattern
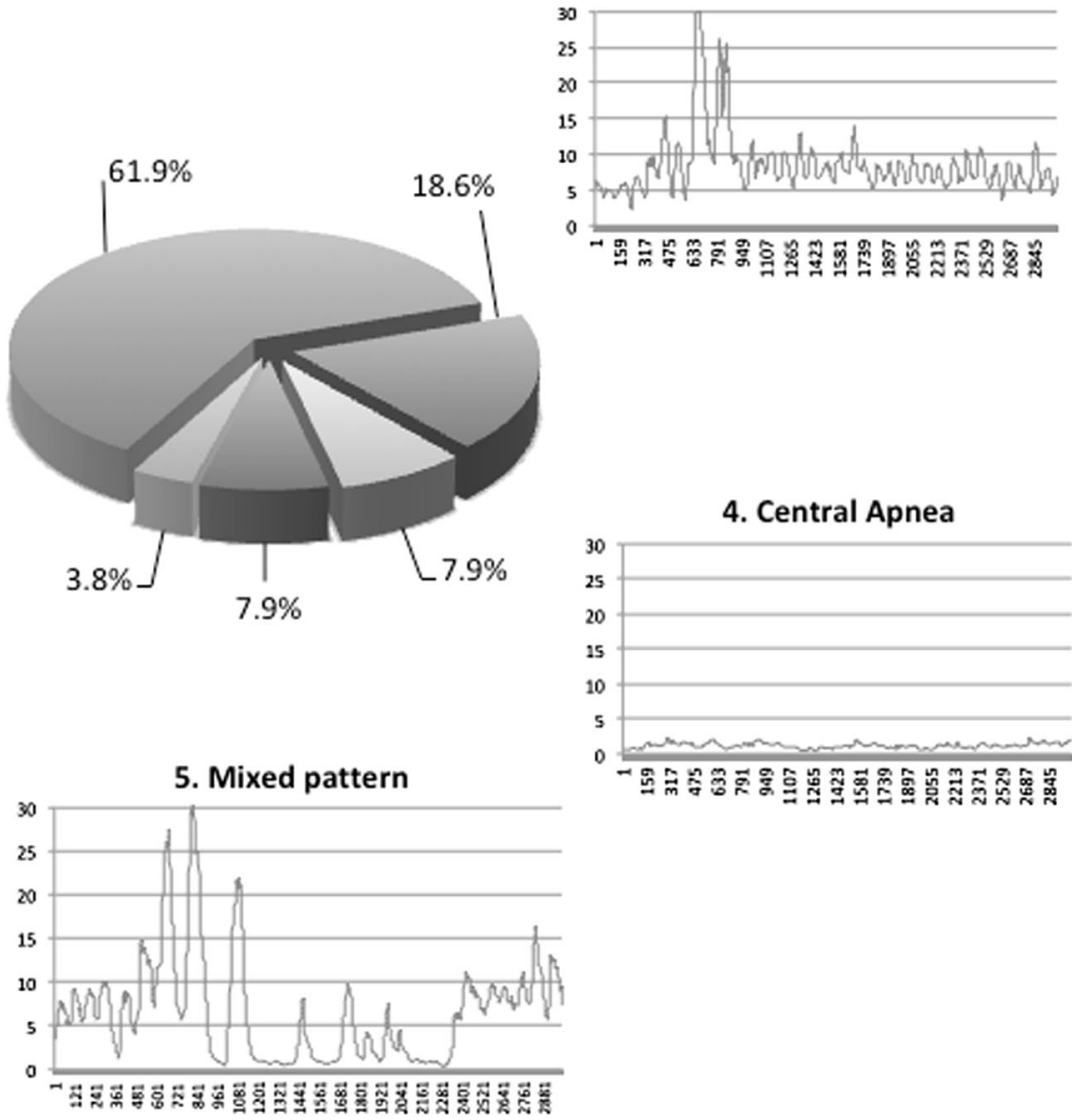

4. Central Apnea

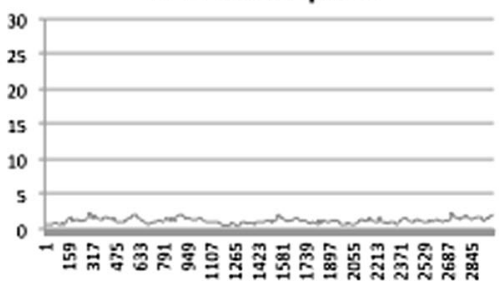

Fig. 3 Distribution of breathing patterns of electrical activity of diaphram (EAdi) in 30-second recordings (EAdi values in microvolts)

average GA four weeks less and an average birth weight $\sim 400 \mathrm{~g}$ lower. In addition, our patients, in contrast to the spontaneously breathing preterm infants of Beck et al., suffered from different forms of pulmonary disease and respiratory failure that, a priori, impose a higher demand on the respiratory system, particularly the diaphragm. A possible explanation for the similarity of the results could be the efficiency of the non-invasive respiratory support applied (PEEP, NAVA level, etc.), compensating the pathophysiogical alterations present in our varied cohort of patients.

Several conditions, such as abdominal distension, lung de-recruitment, pulmonary edema, and acute lung injury, have been shown to increase the tonic activity of inspiratory muscles in animal models [7-10], and there also seems to be a relationship between hypoxia and tonic activity of the diaphragm [11]. As stated above, when alveolar instability and low compliance are present, as could be the case in preterm infants, a higher EAdi Tonic $_{\text {might be necessary to }}$ maintain the end-expiratory lung volume and FRC. In an elegant study, Emeriaud et al. [4] showed that the diaphragm remains partially active during expiration in intubated and mechanically ventilated infants and that removal of PEEP affects this tonic activity. In our study, the PEEP level was established on clinical grounds and might have influenced the $\mathrm{EAdi}_{\mathrm{Min}}$ and $\mathrm{EAdi}_{\mathrm{Tonic}}$ values, but we did not systematically evaluate this relationship. In our NICU, in addition to other clinical criteria, the current standard practice is to increase the PEEP level in a stepwise manner when a patient exhibits a sustained elevation of the EAdi $_{\text {Min }}$ (greater than $5 \mu \mathrm{V}$ ) until it "normalizes". Although reference values are scarce in the neonatal period, in a small cohort of term neonates, Stein et al. found a mean EAdi ${ }_{\text {Min }}$ of $3 \pm 2 \mu \mathrm{V}$ [12], and in ventilated newborns with a median (range) GA of 27 (24-41) weeks, Mally et al. found a median (IQR) EAdi ${ }_{\text {Min }}$ of $2.0(1.5-3.1) \mu \mathrm{V}$ [13].

Other mechanisms may help maintain the lung volume, such as an increase in the respiratory rate, the laryngeal braking of expiratory flow, and deep, prolonged inspirations (sighs) [14-16]. The nRR of our patients could be considered as the upper limit of normal or mild tachypnea, and there was at least one sigh in half of the observations, equivalent to two sighs per minute. This latter finding is similar to that of Beck et al. [1] who reported around one large neural inspiration per minute of observation in healthy preterm babies. Surprisingly, patients in the study exhibited a higher nRR (mean of 73 
Table 2 Electrical activity of diaphragm (EAdi) and neural times in preterm infants assisted with NIV-NAVA

\begin{tabular}{lllllllll}
\hline & EAdi $_{\text {Peak }}(\mu \mathrm{V})$ & EAdi $_{\text {Tonic }}(\mu \mathrm{V})$ & EAdi $_{\text {Phasic }}(\mu \mathrm{V})$ & EAdi $_{\text {Min }}(\mu \mathrm{V})$ & $\mathrm{nTi}(\mathrm{ms})$ & $\mathrm{nTe}(\mathrm{ms})$ & $\mathrm{nTbt}(\mathrm{ms})$ & $\mathrm{nRR}(\mathrm{bpm})$ \\
\hline Mean & $7.5(6.8-10.6)$ & $3.4(3.1-5.3)$ & $4.2(3.7-5.6)$ & $2.5(2.2-4.2)$ & $279(253-285)$ & $764(642-925)$ & $1053(916-1204)$ & $63(51-70)$ \\
$\mathrm{SD}$ & $3.8(3.1-5.6)$ & $2.0(1.3-2.4)$ & $2.4(1.9-4.0)$ & $1.9(1.3-2.4)$ & $41(35-67)$ & $247(147-367)$ & $251(165-375)$ & $13.0(11-16)$ \\
$\mathrm{CV}(\%)$ & $45(41-58)$ & $51(40-67)$ & $53(48-65)$ & $63(53-81)$ & $20(14-24)$ & $28(22-41)$ & $22(17-32)$ & $23(18-27)$ \\
\hline
\end{tabular}

Mean, SD, and CV were calculated for each individual patient. Next, the median (IQR) of the individual means, SD and CV were computed for the whole group of 19 patients evaluated and the results are shown in the table.

See text for abbreviations.

bpm) than did our patients, although the nTi $(278 \mathrm{~ms})$ and $\mathrm{nTe}(867 \mathrm{~ms})$ were close to those of our infant cohort. In any case, it is interesting to note that in both studies, the nTi was apparently shorter than that usually applied for assisted ventilation of the newborn, both invasive and non-invasive. When the $\mathrm{Ti}$ is indirectly estimated based on flow or pressure changes, discrepancies in timing or duration might result in different types of dyssynchrony and patient discomfort [17]. Recently, Mally et al. in the aforementioned group of patients [13], found a median (IQR) nTi of 478 (411-536) ms. However, in the study the nTi was calculated as the time between the onset of EAdi and when it dropped to $70 \%$ of its peak. Of interest, Emeriaud et al. [4] found a significant correlation between the inspiratory EAdi and the EAdi during the first quartile (Q1) of expiration, suggesting that some diaphragmatic motor units may not be deactivated at that moment. Nevertheless, this EAdi during Q1 possibly might be better explained as post-inspiratory activity, presumably responding to vagal messages from lung volume. In a model of newborn piglets, Lawson et al. demonstrated three types of respiratory neurons in the lateral medulla: inspiratory neurons, post-inspiratory neurons, with a short decrementing firing pattern that started immediately after inspiration ended, and expiratory neurons, suggesting a three-phased mechanism of respiratory control [18]. This post-inspiratory activity has been observed in diaphragmatic myograms of neonates [19].

Another important finding of our study is the high variability in all the neural respiratory parameters studied (Table 2). In experimental models, in contrast to modalities that provide constant pressure or volume support, breath-tobreath variability has been shown to improve $\mathrm{V}_{\mathrm{T}}$ distribution, lung recruitment and endogenous surfactant production [20], and lead to better ventilation/perfusion matching and lung mechanics, as well as less inflammation and an eventual reduction in the risk of ventilator-induced lung injury [21]. NAVA allows for continuous adjustment of the $\mathrm{nTi}$, nRR and $\mathrm{V}_{\mathrm{T}}$ (through changes in EAdi $\mathrm{Phasic}_{\text {) }}$ ) according to the variable needs of the patient, reflecting the central respiratory drive. It also allows the patient to perform lung recruitment maneuvers, such as sighs, or prevent lung derecruitment, increasing the EAdi $\mathrm{T}_{\text {Tonic }}$. Whether this variability results in a significant long-term advantage for the individual remains to be determined [22].
Our study has some limitations. The population studied, although representative of the mild to moderate respiratory disease of the preterm infant, was heterogeneous and the periods of observation were brief, the $30 \mathrm{~s}$ of direct recording that allows the software of the equipment. So some selection biases could have influenced our results. Moreover, variability over time was not studied.

In conclusion, the neural breathing patterns of preterm infants with moderate lung disease in need of non-invasive support seem to be quite variable and are characterized by the presence of increased tonic activity during a significant proportion of the respiratory cycles. Central apneas and sighs are common in this group of patients. A synchronized respiratory support system, capable of adapting to the patient's needs, detect apneas and offer rescue ventilation, could result in important clinical benefits. More studies are needed to adequately characterize the neural respiratory patterns and their variation over time in the different respiratory pathologies of the newborn, as well as the longterm potential clinical benefit of neural syncronization.

Acknowledgements The authors thank all the nurses of the Neonatal Intensive Care Unit for their contribution to the collection of data and for their loving care of the patients and their families.

\section{Compliance with ethical standards}

Conflict of interest The authors declare that they have no conflict of interest.

\section{References}

1. Beck J, Reilly M, Grasselli G, Qui H, Slutsky AS, Dunn MS, et al. Characterization of neural breathing pattern in spontaneously breathing preterm infants. Pediatr Res. 2011;70:607-13.

2. Aldrich TK, Sinderby C, McKenzie DK, Estenne M, Gandevia SC. Electrophysiologic techniques for the assessment of respiratory muscle function. American Thoracic Society/European Respiratory Society 2002 ATS/ERS Statement on Respiratory Muscle Testing. Am J Respir Crit Care Med. 2002;166:518-624.

3. Lopes J, Muller NL, Bryan MH, Bryan AC. Importance of inspiratory muscle tone in maintenance of FRC in the newborn. $\mathrm{J}$ Appl Physiol. 1981;51:830-4.

4. Emeriaud G, Beck J, Tucci M, Lacroix J, Sinderby C. Diaphragm electrical activity during expiration in mechanically ventilated infants. Pediatr Res. 2006;59:705-10. 
5. Allo JC, Beck JC, Brander L, Brunet F, Slutsky AS, Sinderby CA. Influence of neurally adjusted ventilatory assist and positive endexpiratory pressure on breathing pattern in rabbits with acute lung injury. Crit Care Med. 2006;34:2997-3004.

6. Barwing J, Ambold M, Linden N, Quintel M, Moerer O. Evaluation of the catheter positioning for neurally adjusted ventilatory assist. Intensive Care Med. 2009;35:1809-14.

7. Meesen NE, van der Grinten CP, Folgering HT, Luijendijk SC. Tonic activity in inspiratory muscles during continuous negative airway pressure. Respir Physiol. 1993;92:151-66.

8. D'Angelo E, Pecchiari M, Acocella F, Monaco A, Bellemare F. Effects of abdominal distension on breathing pattern and respiratory mechanics in rabbits. Respir Physio Neurobiol. 2002;130:293-304.

9. Gunawardena S, Ravi K, Longhurst JC, Kaufman MP, Ma A, Bravo $\mathrm{M}$, et al. Responses of $\mathrm{C}$ fiber afferents of the rabbit airway and lungs to changes in extra-vascular fluid volumen. Respir Physiol Neurobiol. 2002;132:239-51.

10. Ma A, Bravo M, Kappagoda CT. Responses of bronchial C-fiber afferents of the rabbit to changes in lung compliance. Respir Physiol Neurobiol. 2003;138:155-63.

11. Bonora M, Boule M, Gautier H. Diaphragmatic and ventilatory responses to alveolar hypoxia and hypercapnia in conscious kittens. J Appl Physiol (1985). 1992;72:203-10.

12. Stein HM, Wilmoth J, Burton J. Electrical activity of the diaphragm in a small cohort of term neonates. Respir Care. 2012;57:1483-7.

13. Mally PV, Beck J, Sinderby C, Caprio M, Bailey SM. Neural breathing pattern and patient-ventilator interaction during neurally adjusted ventilatory assist and conventional ventilation in newborns. Pediatr Crit Care Med. 2018;19:48-55.
14. Kosch PC, Stark AR. Dynamic maintenance of end-expiratory lung volume in full-term infants. J Appl Physiol. 1984;57:1126-33.

15. Kosch PC, Hutchinson AA, Wozniack JA, Carlo WA, Stark AR. Posterior cricoarytenoid and diaphragm activities during tidal breathing in neonates. J Appl Physiol (1985). 1988;64:1968-78.

16. Alvarez JE, Bodani J, Fajardo CA, Kwiatkowski K, Cates DB, Rigatto H. Sighs and their relationship to apnea in the newborn infant. Biol Neonate. 1993;63:139-46.

17. Parthasarathy S, Jubran A, Tobin MJ. Assessment of neural inspiratory time in ventilator-supported patients. Am J Respir Crit Care Med. 2000;162:546-52.

18. Lawson EE, Richter DW, Bischoff A. Intracellular recordings of respiratory neurons in the lateral medulla of piglets. J Appl Physiol. 1989;66:983-8.

19. Stark AR, Cohlan BA, Waggener TB, Frantz ID 3rd, Kosch PC. Regulation of end-expiratory lung volume during sleep in premature infants. J Appl Physiol. 1987;62:1117-23.

20. Arold SP, Bartolák-Suki E, Suki B. Variable stretch pattern enhances surfactant secretion in alveolar type II cells in culture. Am J Physiol Lung Cell Mol Physiol. 2009;296: L574-81.

21. Spieth PM, Carvalho AR, Pelosi P, Hoehn C, Meissner C, Kasper $\mathrm{M}$, et al. Variable tidal volumes improve lung protective ventilation strategies in experimental lung injury. Am J Respir Crit Care Med. 2009;179:684-93.

22. García-Muñoz Rodrigo F, Urquía Marti L, Galán Henríquez G, Rivero Rodríguez S, Siles Quesada C. Neurally adjusted ventilatory assist increases respiratory variability and improves patient-ventilator synchronisation. Pediatr (Barc). 2016;84:231-2. 